\title{
THE SURFACE- AND SIZE-DEPENDENT ELASTIC MODULI OF NANOSTRUCTURES
}

\author{
JIAN-GANG GUO and YA-PU ZHAO* \\ State Key Laboratory of Nonlinear Mechanics (LNM), \\ Institute of Mechanics, Chinese Academy of Sciences, \\ Beijing 100080, China \\ *yzhao@imech.ac.cn
}

Received 19 December 2006

\begin{abstract}
A theoretical model is presented to investigate the size-dependent elastic moduli of nanostructures with the effects of the surface relaxation surface energy taken into consideration. At nanoscale, due to the large ratios of the surface-to-volume, the surface effects, which include surface relaxation surface energy, etc., can play important roles. Thus, the elastic moduli of nanostructures become surface- and size-dependent. In the research, the three-dimensional continuum model of the nanofilm with the surface effects is investigated. The analytical expressions of five nonzero elastic moduli of the nanofilm are derived, and then the dependence of the elastic moduli is discussed on the surface effects and the characteristic dimensions of nanofilms.
\end{abstract}

Keywords: Size-dependent elastic moduli; surface effects; nanostructures.

\section{Introduction}

Nanostructures in general can be defined as the structures whose at least one of the overall dimensions is in the nanometer range, which include nanofilms, nanobelts, nanowires, nanoparticles, etc. The mechanical properties of nanostructures, such as elastic modulus, hardness, yield stress, etc., are generally different from those at the macroscopic scale. They are often size- and surface-dependent. Thus, it will produce wrong results if the difference is not taken into consideration in the course of design and application.

The elastic moduli are fundamental mechanical properties of materials, which are defined in the theory of continuum mechanics. The elastic moduli are constant for a specified material at macroscopic scale, but it had been shown by a great deal of experiments ${ }^{1-6}$ that they exhibit size effects at nanoscale. The elastic moduli can either increase ${ }^{1-3}$ or decrease ${ }^{4-6}$ when the size of the nanostructures is reduced. The explanation to the phenomena is usually attributed to surface effects, including surface relaxation, surface energy, etc., which play predominant roles at nanoscale due to the large surface-to-volume ratio of nanostructures. Some theoretical models ${ }^{7,8}$ were presented and molecular simulation $^{9-11}$ was done to study the size dependence of elastic moduli taking the surface effects into consideration, but a uniform and extensively acceptable model is still desirable. Considering the effects of the surface relaxation, the present authors ${ }^{12}$ also presented a three-dimensional lattice model to investigate the elastic moduli of nanofilms, and showed that the size effect is dependent on the surface relaxation. Based on the work in Ref. 12, further researches will be made in the paper to study the elastic moduli of nanofilms with the effects of surface relaxation and surface energy.

\footnotetext{
${ }^{*}$ Corresponding author.
} 


\section{The Elastic Moduli of Nanofilm Taking Surface Relaxation and Surface Energy into Consideration}

A three-dimensional simple cubic lattice model of a nanofilm is applied. ${ }^{12}$ The film is nanoscale in the direction of thickness, which is equal to $h$, and infinite in directions of length and width. There are $2 N+1(N=1,2,3, \ldots$,$) layers of atoms along the$ thickness, and the surface relaxation takes place on the outmost atomic layers. The surface relaxation coefficient $k$ is introduced, and the surface atoms expand when the relaxation coefficient $k>1$, and contract when $k<1$. When it deforms, the total energy of the nanofilm is composed of the bulk strain energy and the surface energy of two free surfaces.

$$
U_{\text {tot }}=U_{b}+U_{s}=V_{0} f+2 A \gamma .
$$

Since the lattices are uniform in the $x-y$ plane, we may choose a crystal lattice width in either direction. As a result, the volume $V_{0}=a^{2} h$ and the area $A=$ $a^{2}$, where $a$ is the distance of the nearest neighbors, and $h$ is the thickness of the film: $h=2(N+k-1) a$.

The density of strain energy of the nanofilm is $w=U_{\text {tot }} / V_{0}=f+2 \gamma / h$. So, the elastic moduli of the nanofilms can be derived,

$$
C_{i j k l}=\frac{\partial^{2} w}{\partial \epsilon_{i j} \partial \epsilon_{k l}}=\frac{\partial^{2} f}{\partial \epsilon_{i j} \partial \epsilon_{k l}}+\frac{2}{h} \cdot \frac{\partial^{2} \gamma}{\partial \epsilon_{i j} \partial \epsilon_{k l}},
$$

where the first term at the right-hand side equals the elastic moduli of the bulk crystal, which had been obtained is Ref. 12 ,

$$
B_{i j k l}=\frac{\partial^{2} f}{\partial \epsilon_{i j} \partial \epsilon_{k l}}, \quad(i, j, k, l=1,2,3) .
$$

and the second term is equal to the surface elastic moduli, which can be derived by the Shuttleworth relation ${ }^{13}$ and the molecular dynamics (MD) simulation. ${ }^{10}$

$$
S_{i j k l}=\frac{\partial^{2} \gamma}{\partial \epsilon_{i j} \partial \epsilon_{k l}}, \quad(i, j, k, l=1,2) .
$$

The elastic moduli tensors possess the Voigt symmetries with respect to the exchange of indices: $T_{i j k l}=T_{j i k l}=T_{i j l k}=T_{k l i j}(T=C, B, S)$. So, the number of independent components in the elastic moduli tensors $C_{i j k l}$ reduce to 21 from 81 . Thus the tensor $C_{i j k l}$ can be replaced by $C_{i j}$, and the constitutive relation of the nanofilm can be expressed as $\sigma_{i j}=C_{i j} \epsilon_{j}(i, j=1,2, \ldots, 6)$, where the subscript $i=1,2, \ldots, 6$ corresponds to $x, y, z, x y, y z$, and $x z$, respectively. By Eqs. (2)-(4), five independent nonzero elastic moduli of the nanofilm are derived when the surface relaxation and the surface energy are taken into consideration. And it is shown that the nanofilm is transversely isotropic.

$$
\begin{aligned}
C_{11}= & C_{22}=\frac{1}{h} \cdot\left[(2 N+1) \alpha_{1}+(4 N-1) \alpha_{2}\right. \\
& \left.+\frac{4 \alpha_{4}}{1+k^{2}}+2 S_{1111}\right], \\
C_{12}= & C_{21}=\frac{1}{h} \cdot\left[(2 N+1) \alpha_{2}+2 S_{1122}\right], \\
C_{13}= & C_{31}=C_{23}=C_{32}=\frac{1}{h} \\
& \times\left[(2 N-1) \alpha_{2}+\frac{4 k^{2} \alpha_{4}}{1+k^{2}}\right], \\
C_{33}= & \frac{1}{h} \cdot\left[(2 N-1)\left(\alpha_{1}+2 \alpha_{2}\right)\right. \\
& \left.+2 k^{2} \alpha_{3}+\frac{8 k^{4} \alpha_{4}}{1+k^{2}}\right], \\
C_{44}= & \frac{1}{h} \cdot\left[4(2 N+1) \alpha_{2}+2 S_{1212}\right], \\
C_{55}= & C_{66}=4 C_{13},
\end{aligned}
$$

where $\alpha_{1}$ and $\alpha_{2}$ denote the spring constants of the nearest neighbors and the next-nearest neighbors between the unrelaxed atoms, respectively; $\alpha_{3}$ and $\alpha_{4}$ are those of the nearest neighbors and the next-nearest neighbors between the relaxed atoms, respectively.

The spring constants $\alpha_{i}(i=1,2,3,4)$, can be obtained by calculating the interatomic potential. It is assumed that the Lennard-Jones potential, $u(r)=$ $\epsilon_{b}\left[\left(r_{0} / r\right)^{12}-2\left(r_{0} / r\right)^{6}\right]$, is applied, where $r_{0}$ is the atomic distance in equilibrium, and $\epsilon_{b}$ is the depth of the potential well. Then we can truncate the Taylor expansion at second order: $u(r)=u\left(r_{0}\right)+(1 / 2) \alpha(r-$ $\left.r_{0}\right)^{2}+\cdots+$, where $\alpha=u^{\prime \prime}\left(r_{0}\right)=\left(72 \epsilon_{b}\right) / r_{0}^{2}$ is equivalent to the spring coefficient. So the spring constants $\alpha_{i},(i=1,2,3,4)$ can also be defined by the parameter $\alpha$ as follows:

$$
\begin{aligned}
& \alpha_{1}=\alpha, \quad \alpha_{2}=\alpha / 2, \\
& \alpha_{3}=\alpha / k^{2}, \quad \alpha_{4}=\alpha /\left(1+k^{2}\right) .
\end{aligned}
$$

On the other hand, if only the interaction between the nearest neighbors is considered, the Young's modulus of the bulk crystal can approximately be calculated by Eq. (14): $E=\alpha / r_{0}$. Thus, the five independent nonzero elastic moduli of 
nanofilms can further be rewritten as the following dimensionless forms:

$$
\begin{gathered}
C_{11}^{*}=\frac{C_{11}}{E}=\frac{1}{n} \cdot\left[\beta_{1}+\frac{a^{2} S_{1111}}{36 \epsilon_{b}}\right], \\
C_{12}^{*}=\frac{C_{12}}{E}=\frac{1}{n} \cdot\left[\beta_{2}+\frac{a^{2} S_{1122}}{36 \epsilon_{b}}\right], \\
C_{13}^{*}=\frac{C_{13}}{E}=\frac{\beta_{3}}{n}, \\
C_{33}^{*}=\frac{C_{33}}{E}=\frac{\beta_{4}}{n}, \\
C_{44}^{*}=\frac{C_{44}}{E}=\frac{1}{n} \cdot\left[\beta_{5}+\frac{a^{2} S_{1212}}{36 \epsilon_{b}}\right],
\end{gathered}
$$

where $n$ is the dimensionless thickness of nanofilms, $n=h / a=2(N+k-1)$, and the dimensionless parameters $\beta_{i}(i=1,2,3,4,5)$ are the following forms, respectively.

$$
\begin{gathered}
\beta_{1}=4 N+\frac{1}{2}+\frac{4}{\left(1+k^{2}\right)^{2}}, \\
\beta_{2}=N+\frac{1}{2}, \\
\beta_{3}=N-1+\frac{4 k^{2}}{\left(1+k^{2}\right)^{2}}, \\
\beta_{4}=2(2 N-1)+\frac{8 k^{4}}{\left(1+k^{2}\right)^{2}}, \\
\beta_{5}=2(2 N+1) .
\end{gathered}
$$

\section{Discussion}

It can be found from Eqs. (7a)-(7e) that the dimensionless elastic moduli $C_{11}^{*}, C_{12}^{*}, C_{13}^{*}, C_{33}^{*}$, and $C_{44}^{*}$ are dependent on the thickness of the nanofilms and the surface relaxation coefficient $k$. Besides that, the elastic moduli $C_{11}^{*}, C_{12}^{*}$, and $C_{44}^{*}$ are also dependent on the surface elastic moduli $S_{i j k l}$, which are the functions of the surface energy $\gamma$. The surface elastic moduli $S_{i j k l}$ can be obtained by MD simulation. ${ }^{10}$ In the research, it is assumed that the free surfaces of the nanofilm are (111) crystal faces of $\mathrm{Cu}$. So the surface elastic moduli are ${ }^{10} S_{1111}=-0.123 \mathrm{eV} / \AA^{2}$, $S_{1122}=-0.140 \mathrm{eV} / \AA^{2}$, and $S_{1212}=0.009 \mathrm{eV} / \AA^{2}$, respectively. In addition, the values of interatomic distance $a$ and potential energy $\epsilon_{b}$ in the calculation choose $a=0.2556 \mathrm{~nm}$ and $\epsilon_{b}=0.69 \times 10^{-19} \mathrm{~J}$.

Figures $1-5$ show the variations of the dimensionless elastic moduli with the atomic layer numbers for different relaxation coefficients $k$. They decrease

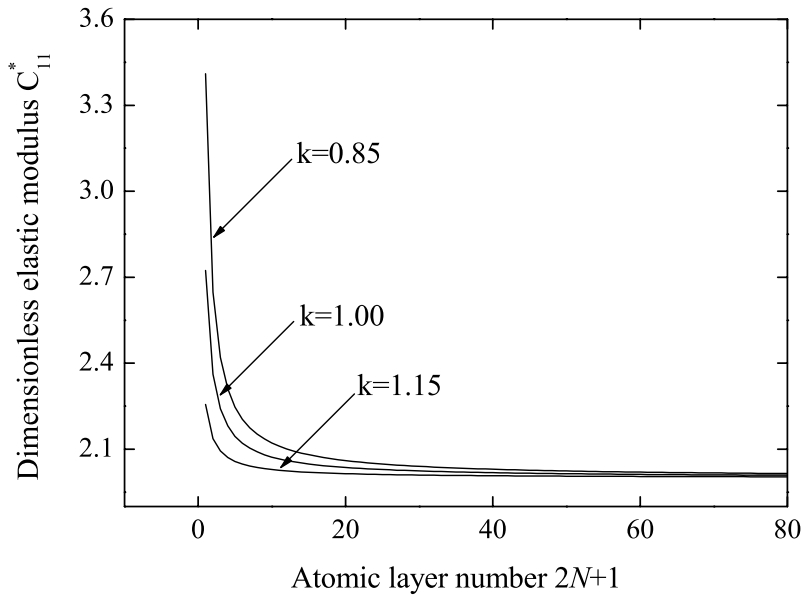

Fig. 1. The variations of elastic modulus $C_{11}^{*}$ with the atomic layer numbers.

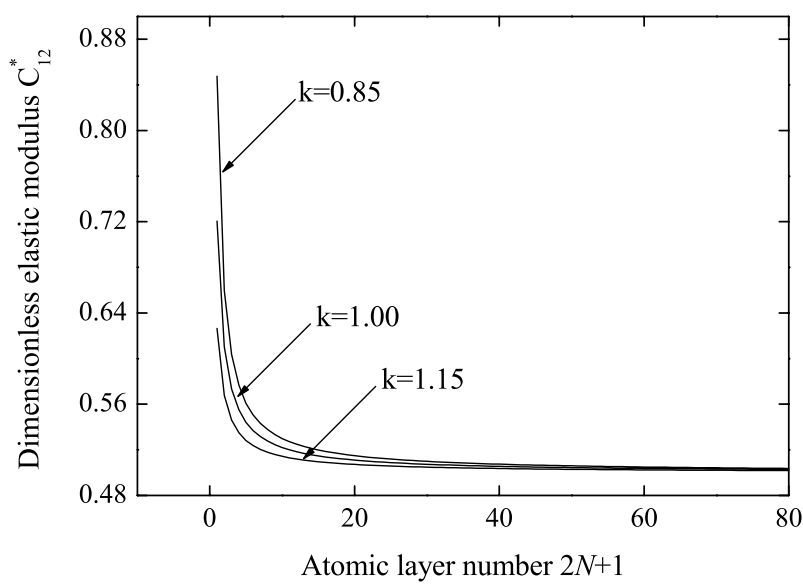

Fig. 2. The variations of elastic modulus $C_{12}^{*}$ with the atomic layer numbers.

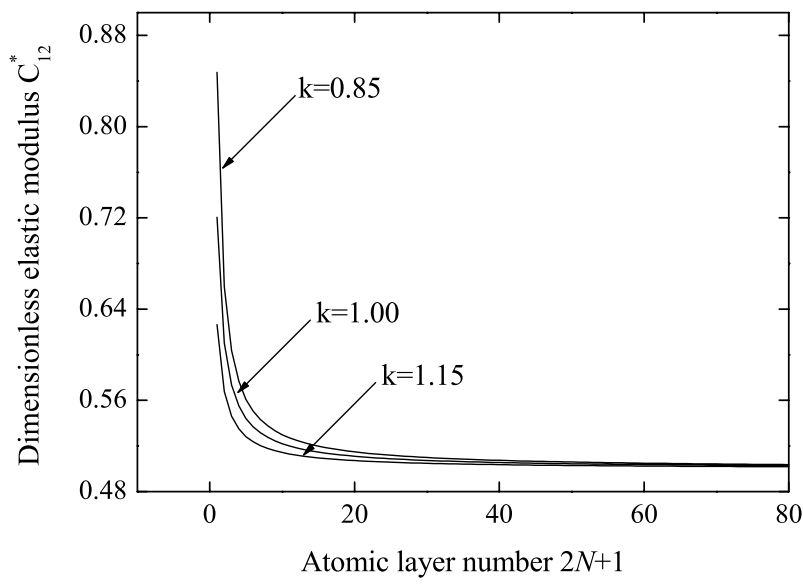

Fig. 3. The variations of elastic modulus $C_{13}^{*}$ with the atomic layer numbers. 


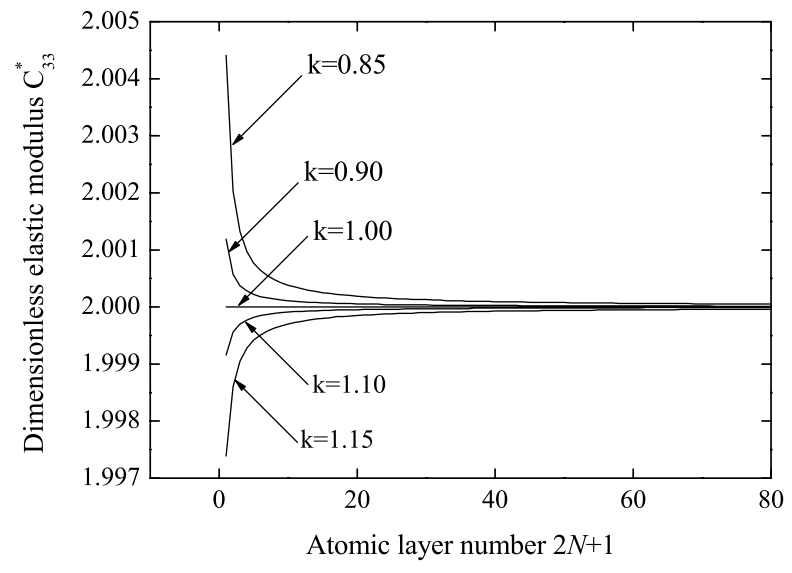

Fig. 4. The variations of elastic modulus $C_{33}^{*}$ with the atomic layer numbers.

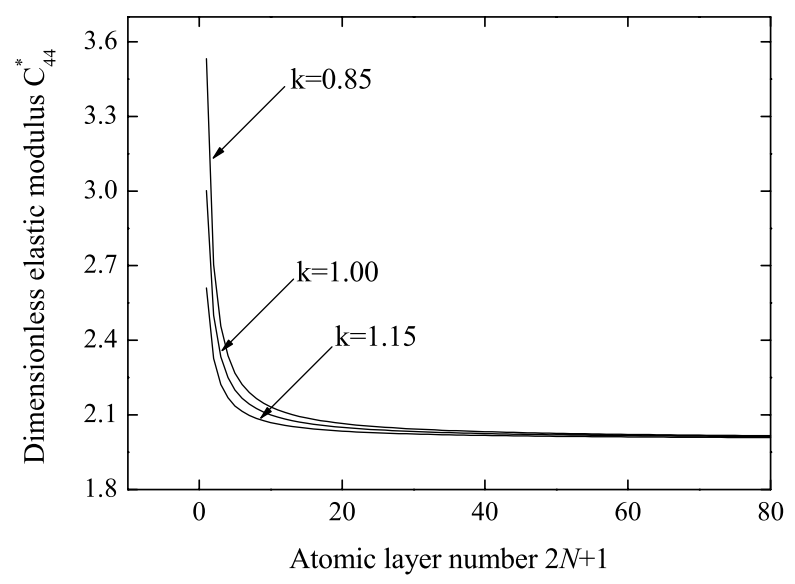

Fig. 5. The variations of elastic modulus $C_{44}^{*}$ with the atomic layer numbers.

or increase with the reduced thickness of nanofilms when the atomic layer numbers are less than 40 , and vary sharply when the atomic layer numbers are less than 20. It can be shown from Figs. 3 and 4 that the elastic moduli $C_{13}^{*}$ and $C_{33}^{*}$ increase with the reduced atomic layer numbers when the relaxation coefficient $k$ is less than $1(k<1)$, and decrease when the relaxation coefficient $k$ is greater than $1(k>1)$. In addition, when the relaxation coefficient $k$ is equal to 1 $(k=1), C_{13}^{*}$ and $C_{33}^{*}$ keep constant. However, for elastic moduli $C_{11}^{*}, C_{12}^{*}$, and $C_{44}^{*}$, besides the surface relaxation, they are also dependent on the surface energy. It can been shown from Figs. 1, 2, and 5 that they always increase, regardless of the values of $k$, when the free surface is the (111) crystal face of $\mathrm{Cu}$, which is a result of the interaction between the surface relaxation and the surface energy.

\section{Conclusion}

Due to the effects of the surface relaxation and surface energy, the nanofilms are transversely isotropic, and there are five independent nonzero elastic moduli. The elastic moduli are size- and surfacedependent. They decrease or increase with the reduced thickness of nanofilms when the atomic layer numbers are less than 40, and vary sharply when the atomic layer numbers are less than 20 . With the decrease of the atomic layer number, elastic moduli $C_{13}^{*}$ and $C_{33}^{*}$ become stiffer when $k<1$, and softer when $k>1$. However, for elastic moduli $C_{11}^{*}, C_{12}^{*}$, and $C_{44}^{*}$, besides the surface relaxation, they are also dependent on the surface energy. When the free surface is the (111) crystal face of $\mathrm{Cu}$, they are always stiffer due to the effects of surface energy.

\section{Acknowledgments}

This work was supported by the National Basic Research Program of China (973 Program, Grant No. 2007CB310504), Distinguished Young Scholar Fund of National Natural Science Foundation of China (NSFC, Grant No. 10225209) and key project from Chinese Academy of Sciences (Grant No. KJCX-SW-L2).

\section{References}

1. S. Cuenot, S. Demoustier-Champagne and B. Nysten, Phys. Rev. Lett. 85 (2000) 1690.

2. S. Cuenot, C. Frétigny, S. Demoustier-Champagne and B. Nysten, Phys. Rev. B 69 (2004) 165410.

3. G. Y. Jing, H. L. Duan, X. M. Sun, Z. S. Zhang, J. Xu, Y. D. Li, J. X. Wang and D. P. Yu, Phys. Rev. B 73 (2006) 235409.

4. M. C. Salvadori, I. G. Brown, A. R. Vaz, L. L. Melo and M. Cattani, Phys. Rev. B 67 (2003) 153404.

5. P. Villain, P. Goudeau, P. O. Renault and K. F. Badwi, Appl. Phys. Lett. 81 (2002) 4365.

6. S. G. Nilsson, X. Borrisé and L. Montelius, Appl. Phys. Lett. 85 (2004) 3555.

7. F. H. Streitz, R. C. Cammarata and K. Sieradzki, Phys. Rev. B 49 (1994) 10699.

8. R. Dingreville, J. Qu and M. Cherkaoui, J. Mech. Phys. Solids 53 (2005) 1827.

9. H. Liang, M. Upmanyu and H. Huang, Phys. Rev. B 71 (2005) 241403.

10. V. B. Shenoy, Phys. Rev. B 71 (2005) 094104.

11. P. Villain, P. Beauchamp, K. F. Badwi, P. Goudeau and P. O. Renault, Script. Mater. 50 (2004) 1247.

12. J. G. Guo and Y. P. Zhao, J. Appl. Phys. 98 (2005) 074306.

13. R. Shuttleworth, Proc. Phys. Soc. A 63 (1950) 444.

14. W. D. Nix and H. Gao, Script. Mater. 39 (1998) 1653. 